\title{
Stability of Charged Shell Supported by a Phantom Energy
}

\author{
Ali Eid ${ }^{1,2}$ \\ ${ }^{1}$ Department of Physics, College of Science, Al Imam Mohammad Ibn Saud Islamic University (IMSIU), Riyadh, KSA \\ ${ }^{2}$ Department of Astronomy, Faculty of Science, Cairo University, Giza, Egypt
}

\section{Email address:}

aeid06@yahoo.com

\section{To cite this article:}

Ali Eid. Stability of Charged Shell Supported by a Phantom Energy. American Journal of Astronomy and Astrophysics. Vol. 3, No. 3, 2015, pp. 50-55. doi: 10.11648/j.ajaa.20150303.14

\begin{abstract}
This paper discusses the evolution of a thin spherically symmetric self gravitating phantom shell around the charged shell. The general equations describing the motion of shell with a general form of equation of state are derived. The different types of space-time $\mathrm{R} \pm$ and $\mathrm{T} \pm$ regions and shell motion are classified depending on the parameters of the problem. The mechanical stability analysis of this spherically symmetric thin shell with charge in Reissner- Nordstrom (RN) to linearized spherically symmetric perturbation about static equilibrium solution is carried out.
\end{abstract}

Keywords: General Relativity, Astrophysics, Cosmology, Phantom Energy

\section{Introduction}

Recent astrophysical observations have confirmed that the Universe is undergoing a phase of accelerated expansion. Evidence of this cosmological expansion, coming from measurements of supernovae of type Ia (SNeIa), Riess and Turner [1], Perlmutter et al. [2] and independently from the cosmic microwave background radiation Bennett et al. [3], Hinshaw [4], shows that the Universe additionally consists of some sort of negative pressure "dark energy". The Wilkinson Microwave Anisotropy Probe (WMAP), designed to measure the $\mathrm{CMB}$ anisotropy with great precision and accuracy, has recently confirmed that the Universe is composed of approximately $70 \%$ of dark energy Bennett et al. [3].

Several candidates representing dark energy have been proposed in the literature, namely, a positive cosmological constant, the quintessence fields, k-essence, h-essence, tachyon models, etc. A simple way to parameterize the dark energy is by an equation of state of the form $\omega=P / \rho$, where

$P$ is the spatially homogeneous pressure and $\rho$ the energy density of the dark energy, Cai and Wang [5]. A value of $\omega \prec-1 / 3$ is required for cosmic expansion, and $\omega=-1$ corresponds to a cosmological constant, Carmeli [6]. One of the peculiar feature of the dark energy is a possibility of the phantom energy equation of state $\rho \succ 0$ and $p \prec 0$ or equivalently $p=-\rho$ ( or $\omega=-1)$. This phantom energy violates the weak energy dominance condition. Matter with the property $\omega \prec-1$ has been denoted "phantom energy".
Recent observational data constrain the range of dark energy by $-1.38 \prec \omega \prec-0.82$ at $95 \%$ confidence level Melchiorri et al. [7]. Rather the supernovae data favor an evolving $\omega$ varying from quintessence $(\omega \succ-1)$ to phantom regime ( $\omega \prec-1$ ), Alam et al. [8]. Further, the extrapolation of WMAP data is best fitted with the notion of phantom energy Caldwell et al. [9].

The most exotic form of dark energy is a phantom energy with $\omega \prec-\frac{1}{3}$, Caldwell [10], for which the weak energy conditions is violated. The exotic nature of phantom energy reveals itself in a number of unusual cosmological consequence (cf. Alcaniz [11], Sushkov [12]). Therefore, one can consider the phantom energy as a possible candidate for exotic matter. It is possible to extend the motion of phantom energy on the case of spherically symmetric space time configurations. Suppose that it is characterized by the equation of state $p=\omega \rho$ with $\omega \prec-\frac{1}{3}$, where $p$ is the negative radial pressure. One of the analytically treatable approach with a fluid self-gravitation is taking into account is the thin shells model. The theory of thin shells in General Relativity was developed by W. Israel [13] and developed then by many authors (see e. g. Berezin et al. [14] for review and references).

Eid [15] discussed a model of radiating phantom shell. The aim of this paper is to describe the thin spherically symmetric phantom shell around a charged body. The paper is organized as follows. In Section 2 the general concepts of 
the dynamics spherically symmetric thin shell are outlined with special attention to Schwarzschild space time. In Section 3 the evolution of charged thin shell with phantom equation of state is analyzed. Outline of a general linearized stability analysis procedure is given in Section 4. A general conclusion is given in Section 5. Throughout the paper we use the units $\hbar$ $=\mathrm{c}=1$.

\section{Dynamics of Thin Shell}

The line element of any spherically symmetric space time can be written in the form

$$
d s^{2}=g_{\alpha \beta} d x^{\alpha} d x^{\beta}=A d t^{2}+2 H d t d q+B d q^{2}+r^{2}(t, q) d \Omega^{2}
$$

Here $t$ and $q$ are the timelike and spacelike coordinates, $A, H$ and $B$ are functions of $t$ and $q$ only, and $r(t, q)$ is the radius of a two dimensional sphere (in the sense that the area of the sphere is $4 \pi r^{2}$ ), and $d \Omega^{2}=d \theta^{2}+\sin ^{2} \theta d \varphi^{2}$, is the line element of the unit sphere. For the given space time the coefficients $A, H$ and $B$ are not uniquely defined. One can transform the line element (1) to the new coordinate system which converses explicitly the spherically symmetric form of the metric

$$
\tilde{t}=\tilde{\tau}(t, q), \quad \tilde{q}=\tilde{q}(t, q)
$$

The radius $r$ is invariant under this transformation. The other very important invariant is

$$
\Delta=\gamma^{\alpha \beta} r_{, \alpha} r_{, \beta}
$$

where $\gamma^{\alpha \beta}$ is inverse to the two-dimensional metric tensor $\gamma_{\alpha \beta}$.

In the flat Minkowskian space time $\Delta=-1$, all the surfaces $r=$ const are time like and $r$ can be chosen as spatial coordinate $q=r$. In the curved space time, $\Delta$ can be positive and negative: (1) The region with $\Delta \prec 0$ is called $R$-region and the radius can be chosen as a radial coordinate $q$. (2) The region with $\Delta \succ 0$ is called $T$-region and the surfaces $r=$ const are spacelike (the normal vector is timelike) and the radius can be chosen as a time coordinate $t$. In $T$-region there is no $\dot{r}=0$ ( where "dot" means a time derivative), hence it must be either $\dot{r} \succ 0$ ( such region of expansion is called $T_{+}-$region) or $\dot{r} \prec 0$ ( such region of contraction is called $T_{-}$-region ). The same holds for $R$-regions. They are divided into two classes which are called $R_{+}$-region with $r^{\prime} \succ 0$ and $R_{-}$-region with $r^{\prime} \prec 0$ (where prime stands for a spatial derivative). These $R$ and $T$ regions are separated by the surfaces $\Delta=0$ which are called the apparent horizons, which can be null, timelike or spacelike apparent horizon.

In this section I give a brief history on the equation of motion of a thin shell. When dealing with the time like spherically symmetric thin shell, adjust the covariant formalism derived by Israel [13] to the case of interest.

Let hypersurface $\Sigma$ divided the whole space time into two parts, "in" and "out", and can connect some special coordinate system called Gauss normal coordinates to this hypersurface $\Sigma$. The line element in these coordinates takes the form

$$
d s^{2}=d \tau^{2}-d n^{2}-r^{2}(\tau, n) d \Omega^{2}
$$

where $d \Omega^{2}=d \theta^{2}+\sin ^{2} \theta d \varphi^{2}$, is the line element of the unit sphere, $\tau$ is the proper time of the observer sitting fixed on $\Sigma$. The coordinate $n$ grows from the "in" to the "out" region in the outer normal direction to the hypersurface $\Sigma$ and $r(\tau, n)$ is the radius of the sphere. The hypersurface is situated at $n=0$ and the intrinsic metric to $\Sigma$ is $d s_{\Sigma}^{2}=d \tau^{2}-R^{2}(\tau) d \Omega^{2}$, where $R(\tau)=r(\tau, 0)$. Keeping in mind that the metric itself is continuous but some of its derivatives make a jump across the shell. The jump of the extrinsic curvature $K_{a b}$ is $\left[K_{a b}\right]=K_{a b}^{\text {out }}-K_{a b}^{i n}$, where a quantity in square brackets stands for the difference of that quantity evaluated on the outer side, say the "out", minus the quantity evaluated on the inner side, the "in" side.

The hypersurface $\Sigma$ represents the history of a surface layer (a singular hypersurface of order one) if $K_{a b}^{o u t} \neq K_{a b}^{i n}$.

The Einstein equation determines the relation between the extrinsic curvature $K_{a b}$ and the three dimensional intrinsic energy momentum tensor $t_{a b}$ is given by the Lanczos equation

$$
\left[K_{a b}\right]=-8 \pi\left(t_{a b}-\frac{1}{2} \operatorname{tg}_{a b}\right)
$$

where $t=t_{a}^{a}$. This relation can be written in the form

$$
t_{a b}=\frac{-1}{8 \pi}\left(\left[K_{a b}\right]-g_{a b}[K]\right)
$$

where $[K]=g^{a b}\left[K_{a b}\right]$ is the trace of the extrinsic curvature. In this case due to spherical symmetry the only nonzero components of $t_{a}^{b}$ are $t_{0}^{0}$ and $t_{2}^{2}=t_{3}^{3} \quad$ [16]. The invariant function $\Delta$ equals $\Delta=R_{, r}^{2}-R_{, n}^{2}$ and

$$
\left.R_{, n}\right|_{\Sigma}=\varepsilon \sqrt{\dot{R}^{2}-\Delta}
$$

where $\varepsilon=+1$ if radii increase in the direction of the outer normal, and $\varepsilon=-1$ if radii decrease. Evidently, $\varepsilon=+1$ in $R_{+}$-region and $\varepsilon=-1$ in $R_{-}$-region. From (3) the components are:

$$
\frac{2 \varepsilon_{\text {in }}}{R} \sqrt{\dot{R}^{2}-\Delta_{\text {in }}}-\frac{2 \varepsilon_{\text {out }}}{R} \sqrt{\dot{R}^{2}-\Delta_{\text {out }}}=8 \pi R t_{0}^{0}
$$




$$
\begin{aligned}
& \frac{2 \varepsilon_{\text {in }}}{R} \sqrt{\dot{R}^{2}-\Delta_{\text {in }}}-\frac{2 \varepsilon_{\text {out }}}{R} \sqrt{\dot{R}^{2}-\Delta_{\text {out }}} \\
& +\frac{\varepsilon_{\text {in }}}{\sqrt{\dot{R}^{2}-\Delta_{\text {in }}}} \ddot{R}-\frac{\varepsilon_{\text {out }}}{\sqrt{\dot{R}^{2}-\Delta_{\text {out }}}} \ddot{R}+ \\
& \frac{\varepsilon_{\text {in }}}{2 R \sqrt{\dot{R}^{2}-\Delta_{\text {in }}}}\left(1+\Delta_{\text {in }}\right)-\frac{\varepsilon_{\text {out }}}{2 R \sqrt{\dot{R}^{2}-\Delta_{\text {out }}}} \\
& \left(1+\Delta_{\text {out }}\right)+4 \pi R\left({ }^{\text {out }} T_{n}^{n}-{ }^{\text {in }} \mathrm{T}_{n}^{n}\right)=8 \pi t_{2}^{2}
\end{aligned}
$$

The continuity equation for the energy-momentum tensor is transformed to

$$
\frac{d t_{0}^{0}}{d \tau}+\frac{2 \dot{R}}{R}\left(t_{0}^{0}-t_{2}^{2}\right)+\left({ }^{\text {out }} \mathrm{T}_{0}^{n}-{ }^{i n} \mathrm{~T}_{0}^{n}\right)=0
$$

This equation is a differential consequence of the first two ones.

In this paper the space-time inside the shell will be the Reissner-Nordstrom (RN) metric and outside the shell is the Schwarzschild metric, then the equations (4), (5) and (6) becomes

$$
\begin{gathered}
\varepsilon_{\text {in }} \sqrt{\dot{R}^{2}+F_{\text {in }}}-\varepsilon_{\text {out }} \sqrt{\dot{R}^{2}+F_{\text {out }}}=4 \pi R t_{0}^{0} \equiv \frac{M}{R} \\
\frac{2 \varepsilon_{\text {in }}}{R} \sqrt{\dot{R}^{2}-\Delta_{\text {in }}}-\frac{2 \varepsilon_{\text {out }}}{R} \sqrt{\dot{R}^{2}-\Delta_{\text {out }}}+ \\
\frac{\varepsilon_{\text {in }}}{\sqrt{\dot{R}^{2}-\Delta_{\text {in }}}} \ddot{R}-\frac{\varepsilon_{\text {out }}}{\sqrt{\dot{R}^{2}-\Delta_{\text {out }}}} \ddot{R}+ \\
\frac{\varepsilon_{\text {in }}}{2 R \sqrt{\dot{R}^{2}-\Delta_{\text {in }}}}\left(1+\Delta_{\text {in }}\right)-\frac{\varepsilon_{\text {out }}}{2 R \sqrt{\dot{R}^{2}-\Delta_{\text {out }}}} \\
\left(1+\Delta_{\text {out }}\right)+4 \pi R\left({ }^{\text {out }} T_{n}^{n}-{ }^{\text {in }} \mathrm{T}_{n}^{n}\right)=8 \pi t_{2}^{2} \\
\dot{t}_{0}^{0}+\frac{2 \dot{R}}{R}\left(t_{0}^{0}-t_{2}^{2}\right)=0
\end{gathered}
$$

where

$$
\begin{gathered}
\Delta_{\text {out }}=-F_{\text {out }}=-1+\frac{2 m_{\text {out }}}{R} \\
\Delta_{\text {in }}=-F_{\text {in }}=-1+\frac{2 m_{\text {in }}}{R}-\frac{q}{R^{2}}
\end{gathered}
$$

and $Q$ is the charge of the shell in the interior space. If $Q^{2} \succ m_{i n}^{2}$ then the metric is non-singular everywhere except at the curvature or the irremovable singularity at $\mathrm{R}=0$. Also if $Q^{2} \leq m_{i n}^{2}$ then the function $F_{i n}(R)$ has two real roots given by $R_{h \pm}=m_{i n} \pm \sqrt{m_{i n}^{2}-Q^{2}}$

These roots physically represent the apparent horizons of the RN black hole. The two horizons are termed the inner $R_{h-}$ and the outer $R_{h+}$. The outer horizon is effectively called the event horizon while the inner one is called the Cauchy horizon of the black hole.

Note that if $Q^{2}=m_{i n}^{2}$, then it represents an extreme RN black hole while if $Q^{2} \succ m_{i n}^{2}$, it yields a naked singularity at $\mathrm{R}$ $=0$, Hawking [17], Poisson [18]. If we take the outer manifold with mass $\mathrm{m}_{\text {out }}$, and the inner one with mass $\mathrm{m}_{\text {in }}=0$ and charge $\mathrm{Q}=0$, it represent, shell around vacuum (bubbles).

\section{Dynamics of Phantom Shell}

The equation of state describing phantom energy in cosmology is usually taken as $p=\omega \rho$ where $\omega \prec-1$ and $p$ is a negative spatially homogeneous pressure. By analogy, it is possible to use the same equation of state for a spherically symmetric distribution of phantom energy but with $p$ is the negative radial pressure and written in the form $p=-k \rho$ with $k \succ 1$. Consider a simple linear equation of state by relation

$$
\mathrm{t}_{0}^{0}=k t_{2}^{2}
$$

In the phantom case $k \succ 1$. The solution of (9) is

$$
t_{0}^{0}=C R^{2(k-1)}
$$

where $C$ is a constant denoted to the shell power. Using equation (7) and taking into account $\left(\varepsilon_{\text {in }}=-1, \varepsilon_{\text {out }}=+1\right)$ one gets the following two equations:

$$
\begin{array}{r}
\left.\dot{R}^{2}=-1+\frac{1}{R}\left\{\left(m_{\text {in }}+m_{\text {out }}\right)+\frac{\delta L^{2}}{4 U}+U-\frac{Q^{2}}{2 R}\right)\right\} \\
\ddot{R}=-\frac{1}{2 R^{2}}\left\{\left(m_{\text {in }}+m_{\text {out }}\right)+k \frac{\delta m^{2}}{U}-2(2 k-1) U-\frac{Q^{2}}{R}+Q^{2} H\right\}
\end{array}
$$

where

$$
H=(1+4 k) \frac{\delta m}{4 U R}+(1+2 k) \frac{Q^{2}}{8 U R^{2}}, \quad U=4 \pi^{2} C^{2} R^{4 k-1}
$$

and

$$
L \equiv \frac{Q^{2}}{2 R}+\left(m_{\text {out }}-m_{\text {in }}\right)=\frac{Q^{2}}{2 R}+\delta m
$$
be

The sign conditions of the equation of motion of shell will

$$
\begin{aligned}
& \varepsilon_{\text {in }}=\operatorname{sign}\left[\delta m+8 \pi^{2} R^{3}\left(t_{0}^{0}\right)\right] \\
& \varepsilon_{\text {out }}=\operatorname{sign}\left[\delta m-8 \pi^{2} R^{3}\left(t_{0}^{0}\right)\right]
\end{aligned}
$$

where $\delta m=m_{\text {out }}-m_{\text {in }}$. In the case of $\delta m \prec 0$ the shell evolves under horizons and cannot reach a distant observer living in $R_{+}$-region. But in the case of $\delta m \succ 0$, the shell can show itself in $R_{+}$-region. When the function $\dot{R}^{2}$ has roots, it is possible to represent both the finite and infinite 
motion. The change of sign of the acceleration $\ddot{R}$ in (13) occurs when $\ddot{R}=0$. This corresponds to the quadratic equation whose positive root is

$$
U_{0}=\frac{1}{4(2 k-1)}\left\{\left(m_{\text {out }}+m_{\text {in }}-\frac{Q^{2}}{R}\right)+\sqrt{W}\right\}
$$

where

$$
\begin{aligned}
& W=\left\{4 m_{\text {out }} m_{\text {in }}+(4 k-1)^{2} \delta m^{2}+4 k^{2} \frac{Q^{4}}{R^{2}}\right. \\
& \left.+\frac{4 Q^{2}}{R}\left[-m_{\text {out }}+(4 k-1) k \delta m\right]\right\}^{\frac{1}{2}}
\end{aligned}
$$

It is convenient to define the parameter space of the problem using $m_{\text {in }}, m_{\text {out }}, k \succ \frac{1}{4}, Q$ and $R$ as free parameters.

\section{Linearized Stability Analysis}

Rearranging equation (12) into the form

$$
\left.\dot{R}^{2}=-1+\frac{1}{R}\left\{\left(m_{\text {in }}+m_{\text {out }}\right)+\frac{\delta L^{2}}{4 U}+U-\frac{Q^{2}}{2 R}\right)\right\}
$$

where $U=4 \pi^{2} C^{2} R^{4 k-1}, C$ is a power constant and $k \succ 1$. This equation can be written in the dynamical form

$$
\dot{R}^{2}+V(R)=0
$$

with the potential given by

$$
V(R)=1-\frac{1}{R}\left\{\left(m_{\text {in }}+m_{\text {out }}\right)+\frac{\delta m^{2}}{4 U}+U+\frac{Q^{2}}{2 R}\left(\frac{Q^{2}}{8 R U}+\frac{\delta m}{2 U}-1\right)\right\}
$$

The factor $F(R)$ and $G(R)$ introduced for computational convenience, are defined by

$$
\begin{gathered}
F(R)=1-\frac{1}{R}\left(m_{\text {in }}+m_{\text {out }}\right) \\
G(R)=\frac{\left(m_{\text {out }}-m_{\text {in }}\right)}{R}
\end{gathered}
$$

So that the potential $V(R)$ takes the form

$$
\begin{aligned}
& V(R)=F(R)-4 \pi^{2} C^{2} R^{2(2 k-1)} \\
& +\frac{q^{2}}{2 R^{2}}-\frac{R}{2 U}\left(\frac{G^{2}}{2}+\frac{Q^{2} G}{2 R^{2}}+\frac{Q^{4}}{8 R^{4}}\right)
\end{aligned}
$$

From equation (14) and $M=4 \pi R^{2} t_{0}^{0}=4 \pi C R^{2 k}$, $U=\pi C M R^{2 k-1}$, equation (20) takes the form

$$
V(R)=F(R)-\left(\frac{M}{2 R}\right)^{2}-\left(\frac{R G}{M}\right)^{2}+\frac{Q^{2}}{M^{2}}\left(\frac{M^{2}}{2 R^{2}}-\frac{Q^{2}}{4 R^{2}}-G\right)
$$

the stable solution at $R=R_{0}$, consider a Taylor expansion of $V(R)$ around $R_{0}$ to second order, provides

$$
\begin{aligned}
& V(R)=V\left(R_{0}\right)+V^{\prime}\left(R_{0}\right)\left(R-R_{0}\right) \\
& +\frac{1}{2} V^{\prime \prime}\left(R_{0}\right)\left(R-R_{0}\right)^{2}+O\left[\left(R-R_{0}\right)^{3}\right]
\end{aligned}
$$

where the prime denotes a derivative with respect to $R$. The first and second derivatives of $V(R)$ are given by

$$
\begin{aligned}
& V^{\prime}(R)=F^{\prime}(R)-2\left(\frac{M}{2 R}\right)\left(\frac{M}{2 R}\right)^{\prime} \\
& -2\left(\frac{R G}{M}\right)\left(\frac{R G}{M}\right)^{\prime}+\frac{Q}{R}\left(\frac{Q}{R}\right)^{\prime}-D^{\prime} \\
& V^{\prime \prime}=F^{\prime \prime}-2\left[\left(\frac{M}{2 R}\right)^{\prime}\right]^{2}-2\left(\frac{M}{2 R}\right)\left(\frac{M}{2 R}\right)^{\prime \prime} \\
& -2\left(\frac{R G}{M}\right)\left(\frac{R G}{M}\right)^{\prime \prime}-D^{\prime \prime}+\mathrm{I}
\end{aligned}
$$

where

$$
D=\frac{Q^{2}}{M^{2}}\left(G+\frac{Q^{2}}{4 R^{2}}\right), \quad I=\left(\frac{Q}{R}\right)^{\prime 2}+\left(\frac{Q}{R}\right)\left(\frac{Q}{R}\right)^{\prime \prime}-2\left(\frac{R G}{M}\right)^{\prime 2} .
$$

Evaluated at the static solution $\left(R=R_{0}\right)$ and through a long calculation, I find that $V\left(R_{0}\right)=0$ and $V^{\prime}\left(R_{0}\right)=0$. From the condition $V^{\prime}\left(R_{0}\right)=0$, one extracts the following useful equilibrium relationship

$$
\begin{aligned}
& \left(\frac{M}{2 R_{0}}\right)^{\prime}=\left(\frac{R_{0}}{M}\right)\left\{F^{\prime}(R)-2\left(\frac{R_{0} G}{M}\right)\left(\frac{R_{0} G}{M}\right)^{\prime}\right. \\
& +\frac{Q}{R_{0}}\left(\frac{Q}{R_{0}}\right)^{\prime}-D^{\prime}\left(R_{0}\right)
\end{aligned}
$$

So that,

$$
\dot{R}^{2}=-\frac{1}{2} V^{\prime \prime}\left(R_{0}\right)\left(R-R_{0}\right)^{2}+O\left[\left(R-R_{0}\right)^{3}\right] .
$$

If $V^{\prime \prime}\left(R_{0}\right) \prec 0$ is verified, then the potential $V(R)$ has a local maximum at $R_{0}$, where a small perturbation in the surface radius will produce an irreversible contraction or expansion of the shell. Therefore, the solution is stable if and only if $V(R)$ has a local minimum at $R_{0}$ and $V^{\prime \prime}\left(R_{0}\right) \succ 0$ is verified. The latter stability condition takes the form

$$
\left(\frac{M}{2 R}\right)\left(\frac{M}{2 R}\right)^{\prime \prime} \prec \Phi-\Psi
$$

where $\Psi$ is defined as

$$
\Phi=\frac{F^{\prime \prime}}{2}-\left(\frac{R G}{M}\right)^{\prime 2}-\left(\frac{R G}{M}\right)\left(\frac{R G}{M}\right)^{\prime \prime}+\left(\frac{Q}{R}\right)^{\prime 2}+\frac{Q}{R}\left(\frac{Q}{R}\right)^{\prime \prime}-D^{\prime \prime}
$$

where $M$ is the surface mass of the shell. Linearized around 
and $\Psi=\left(\frac{M}{2 R}\right)^{\prime 2}$. Also, equation (26) can be written in the form

$$
D^{\prime \prime} \prec F^{\prime \prime}-2\left(\frac{M}{2 R}\right)^{\prime 2}-2\left(\frac{R G}{M}\right)\left(\frac{R G}{M}\right)^{\prime \prime}-2\left(\frac{M}{2 R}\right)\left(\frac{M}{2 R}\right)^{\prime \prime}+I
$$

From $V^{\prime}\left(R_{0}\right)=0$ we get

$$
\begin{aligned}
& R_{0}\left(m_{\text {out }}+m_{\text {in }}\right)-8 \pi^{2} C^{2}(2 K-1) R_{0}^{4 k} \\
& +\frac{k}{4 \pi^{2} C^{2}} \delta m^{2} R_{0}^{-4 k+2}-\frac{Q^{2}}{16 \pi^{2} C^{2}} E=0
\end{aligned}
$$

where

$$
\begin{gathered}
E=(-1-4 k) \delta m R_{0}^{-4 k+1}+\frac{1}{2} Q^{2}(-1-2 k) R_{0}^{-4 k}+16 \pi^{2} C^{2}, \\
C_{ \pm}^{2}=\frac{R_{0}^{-4 k+1}}{16 \pi^{2}(2 k-1)}\left\{\left(m_{\text {out }}+m_{\text {in }}\right)-\frac{Q^{2}}{R}\right. \\
\pm \sqrt{\left.\frac{4 Q^{2}}{R^{2}} \Theta+4 m_{\text {out }} m_{\text {in }}+(4 k-1)^{2} \delta m^{2}\right\}}
\end{gathered}
$$

and

$$
\Theta=Q^{2} k^{2}+R\left[m_{\text {out }}+\left(4 k^{2}-k-1\right) \delta m\right] .
$$

If $Q=0$, then

$C_{ \pm}^{2}=\frac{R_{0}^{-4 k+1}}{16 \pi^{2}(2 k-1)}\left\{\left(m_{\text {out }}+m_{\text {in }}\right) \pm \sqrt{4 m_{\text {out }} m_{\text {in }}+(4 k-1)^{2} \delta m^{2}}\right\}$

For $V^{\prime \prime}\left(R_{0}\right)=0$,

$$
a \frac{Q^{4}}{M^{4}}+b \frac{Q^{2}}{M^{2}}+c=0
$$

where

$$
\begin{gathered}
a=\frac{M^{2}}{2 R^{4}}(2 k-1)(4 k+3), \\
b=\frac{3}{R^{4}} M^{2}-\frac{2}{R^{3}}(2 k+1)(4 k+1) \delta m, \\
c=\frac{M^{2}}{R^{4}}\left\{-\frac{1}{2}(2 K-1)(4 k-3)+\frac{4 k R^{2}}{M^{4}}(R\right. \\
\left.-2 k-1) \delta m^{2}-\frac{2 R}{M^{2}}\left(m_{\text {out }}+m_{\text {in }}\right)\right\}
\end{gathered}
$$

then,

$$
\frac{|Q|}{M} \succ \frac{1}{(2 k-1)(4 k+3)}\left\{-3+\frac{2 R}{M^{2}}(2 K+1)(4 K+1) \pm \sqrt{\Omega}\right\}
$$

where

$$
\begin{aligned}
& \Omega=-64 k^{3}(k-1)+2\left(10 k^{2}-18 k+9\right)-\frac{8 R m_{\text {out }}}{M^{2}}\left(8 k^{2}\right. \\
& +8 k+3)+\frac{16 R k m_{\text {in }}}{M^{2}}(8 k+5)+\Gamma \delta m^{2}
\end{aligned}
$$

and

$$
\Gamma=\frac{4 R^{2}}{M^{4}}\left[-2 k R\left(8 k^{2}+2 k-3\right)+4 k^{2}\left(24 k^{2}+30 k+11\right)+6 k+1\right]
$$

\section{Conclusions}

As the Universe is probably constituted of approximately $70 \%$ of null energy condition violating phantom energy, this cosmic fluid may be used as a possible source to theoretically construct thin shell. In fact, it was found that infinitesimal amounts of phantom energy may support thin shell. We have modeled phantom shell by matching an interior RN geometry, satisfying the equation of state $P=\omega \rho$ with $\omega \prec-1$, to an exterior vacuum solution at a finite junction interface.

The matter is that in GR any type of energy is gravitating, not only energy density but also the tension and pressure are gravitating. The pressure plays a twofold role. The positive pressure causes both repulsion and attraction, the attraction is due to its contribution to the gravitating source. The negative pressure leads to the gravitational repulsion. Hence, the phantom shell is even more repulsive.

In the case of $\delta m \succ 0$ the distance observer may see the shell but cannot register the energy flux of the shell. The stability analysis of this phantom shell to linearized spherically symmetric perturbation about static equilibrium solution is carried out.

\section{References}

[1] A. Riess, M.S. Turner, arXiv: astro-ph:0106051, 2001.

[2] S. Perlmutter, M. S.Turner, M. White, Phys. Rev. Lett. 83, 670, 1999.

[3] C. L. Bennett, M. Halpern, G. Hinshaw, N. Jarosik, A. Kogut, M. Limon, S.S. Meyer, L. Page, et al., Astrophys. J. Suppl. 148, $1,2003$.

[4] G. Hinshaw, et al., arXiv: astro - ph/0302217, 2003.

[5] R. Cai, A. Wang, arXiv: hep - th/0411025, 2004.

[6] M. Carmeli, arXiv: astro - ph/0111259, 2001.

[7] A. Melchiorri, et al., Phys. Rev. D. 68, 043509, 2003.

[8] U. Alam, et al., Mon. Not. R. Astron. Soc. 354, 275, 2004.

[9] R. R. Caldwell, et al., Phys. Rev. Lett. 91, 071301, 2003.

[10] R.R. Caldwell, Phys. Lett. B. 545, 23, 2002.

[11] J.S. Alcaniz, Phys. Rev. D. 69, 083521, 2004.

[12] S. Sushkov, gr-qc. 0502084, 2005.

[13] W. Israel, Nuovo Cimento B, 44, 1, 1966. 
[14] V.A. Berezin, V.A. Kuzmin and I.I. tkachev, Phys. Rev. D., 36, 2919, 1987.

[15] A. Eid, EJTP 3, No. 13, 109, 2006.

[16] H. Sato, Prog. Theor. Phys., 76, 1250, 1986.
[17] S.W. Hawking, G.F.R. Ellis, The large scale structure of space-time, Cambridge University Press, 1973.

[18] E. Poisson, A relativist's tool kit: the mathematics of black hole mechanics, Cambridge University Press, 2004. 